\title{
A finite element analysis for predicting outcomes of cemented total knee arthroplasty
}

\author{
NICOLAE GHEORGHIU ${ }^{1,2 *}$, BOGDAN SOCEA ${ }^{3,4}$, MIHAI C.T. DIMITRIU ${ }^{5,6}$, \\ NICOLAE BACALBASA ${ }^{5,7}$, GABRIEL STAN $^{1,2^{*}}$ and HORIA ORBAN ${ }^{1,2^{*}}$ \\ ${ }^{1}$ Department of Orthopedics, 'Carol Davila' University of Medicine and Pharmacy, 020021 Bucharest; \\ ${ }^{2}$ Department of Orthopedics, Elias Emergency Clinical Hospital, 011416 Bucharest; ${ }^{3}$ Department of Surgery, \\ 'Carol Davila' University of Medicine and Pharmacy, 020021 Bucharest; ${ }^{4}$ Department of Surgery, \\ 'Sf. Pantelimon' Emergency Hospital, 021659 Bucharest; ${ }^{5}$ Department of Obstetrics and Gynecology, \\ 'Carol Davila' University of Medicine and Pharmacy, 020021 Bucharest; ${ }^{6}$ Department of Obstetrics and Gynecology, \\ 'Sf. Pantelimon' Emergency Hospital, 021659 Bucharest; ${ }^{7}$ Department of Obstetrics and Gynecology, \\ 'Dr. I. Cantacuzino' Clinical Hospital, 020475 Bucharest, Romania
}

Received October 9, 2020; Accepted November 11, 2020

DOI: $10.3892 /$ etm.2021.9698

\begin{abstract}
The study was designed to assess the validity of a finite element analysis for predicting the behavior of cemented knee implant used in total knee arthroplasty (TKA), for different mechanical loads, and correlation with clinical outcomes of this procedure. We conducted computational simulations using finite element analysis of two situations: i) The ideal prosthetic component positioning; and ii) variable varus tibial malposition, but with a balanced knee. A total of 80 cemented TKAs performed on 70 patients were divided into two groups. Patients from one group required secondary asymmetric tibial recut for balancing the prosthetic knee and patients from the other group, did not. In regards to the results, we observed no differences upon analysis of the postoperative results of the Knee Society Score (KSS), the angle between the femur and tibia, the range of motion and frontal laxity between groups. The finite element analysis showed that in a $3^{\circ}$ varus inclination of the joint interline, but with a balanced knee, the maximum contact stress, measured on the tibial plateau surface, increased by $11 \%$ compared to the value of mechanical alignment. In conclusion, analysis of the computational model using finite elements showed predictable results of cemented TKA for the different situations of mechanical loads.
\end{abstract}

Correspondence to: Lecturer Bogdan Socea, Department of Surgery, 'Carol Davila' University of Medicine and Pharmacy, 37 Dionisie Lupu Street, 020021 Bucharest, Romania

E-mail: bogdan.socea@umfcd.ro

${ }^{*}$ Contributed equally

Key words: biomedical computing, polyethylene stress, clinical trials, orthopedic procedures, surgery

\section{Introduction}

Finite element analysis can be used to calculate the tensile stress on a polyethylene tibial insert and von Mises stress. Polyethylene (PE) wear leads to a process known as 'particle disease' a factor of failure of the procedure. Total knee arthroplasty (TKA) has become a routine intervention for treating late stage osteoarthritis worldwide. Its success, in terms of function and survivorship, costs, days of hospitalization, depends on several factors, including component alignment and ligament balance (1). The ideal conditions for success of TKA are still a matter of debate in the literature. On the one hand, most researchers agree that, for better survivorship, the overall alignment should be neutral or slightly valgus and the tibial component must be positioned at a right angle to the tibial mechanical axis $(2,3)$. On the other hand, there are studies which have demonstrated no difference in terms of survival between groups of neutrally aligned and other then neutrally aligned knees after TKA (4). These authors argue that the mechanical axis goal of $0 \pm 3^{\circ}$ is not enough to predict the durability of modern TKA implants (4). Furthermore, an additional issue of TKA is post-surgery patient satisfaction. Studies report that patient satisfaction ranges between 75 and $92 \%$, even in the case of knees with neutral alignment of components. For example, a systematic review of studies on post-TKA patient satisfaction published between 2000 and 2012 showed only $85 \%$ satisfaction (5). The new concept of kinematic knee alignment arose as a response to the issues discussed above. Kinematic knee alignment aims to improve the functionality of the patient knee and to control pain, with minimal surgical involvement, centered on ligament balance (6). Despite the widespread view that neutral alignment is a condition for TKA success, kinematic knee alignment does not always ensure neutral overall alignment and tibial alignment. These findings highlight the combined importance of both alignment and ligament balance for TKA outcomes. In some cases, especially in severe varus knees, standard 
bone cuts and safety ligament releases may not be enough for balancing the TKA. In such cases, secondary asymmetric tibial resection may be necessary to achieve balance, although overall alignment may not remain neutral. Good knowledge of contact pressures and pressure areas in TKA is the key to success for the knee implant. It has been shown that higher contact stress on the tibial bearing component is associated with more severe damage in the tibial components (6). The present study assessed the validity of a finite element analysis for predicting behavior over time of a cemented knee implant used in TKA, for different mechanical loads, and the correlation with clinical outcomes of this procedure.

\section{Patients and methods}

Our study took place between 2008 and 2015. A total of 80 TKAs were performed on 70 patients. The patients were split in two groups. Whereas in group 1 of 50 TKAs, secondary asymmetric tibial recut was not necessary, group 2 of 30 TKAs, needed secondary asymmetric tibial recut for balancing. Out of the 70 patients, the sex ratio was 45:25 in favor of women. At the moment of TKA, the mean age of the patients was 65.5 . The mean body mass index (BMI) was $24.9 \mathrm{~kg} / \mathrm{m}^{2}$. Group 1 consists of 25 female and 18 male patients; mean age 62.5 years, mean BMI $26 \mathrm{~kg} / \mathrm{m}^{2}$. Regarding group 2, it included 27 patients, of which 20 were female and 7 men. Participants in group 2 had a mean age of 61.4 years and a mean BMI of $23.2 \mathrm{~kg} / \mathrm{m}^{2}$. All surgical procedures were performed by the same senior surgeon. For all cases, the same type of posterior-stabilized cemented knee prosthesis (Zimmer-Nexgen) was utilized. All osteophytes were removed. Next, medial ligament release was performed, using a subperiosteal osteotome. For the patients in group 1, these actions alone led to a balanced knee, thus we did not need further procedures. For the patients in group 2, a further asymmetrical tibial coronal recut was needed, because of the tightness in flexion and extension of the medial compartment. The surgeon removed an extra slice of bone, 2-mm thickness, from the medial tibial plateau. After this artifice, the knee became balanced, with equal extension and flexion gaps. Recovery began immediately after surgery, with alternating placement of the knee in the flexion and extension positions. Postoperative control was conducted at 6 weeks, 3 months, 6 months, and 1 year periods, and then, once yearly. The mean follow-up period for the patients was 5 years ( \pm 3 years). The range of motion, stability to varus-valgus stress and Knee Society Score (KSS) (7) were noted for all patients, preoperatively and during each postoperative visit. Standing standard AP and a lateral view X-ray exam of the knee was conducted preoperatively and postoperatively. We also conducted computational simulations using finite element analysis of two situations: i) The ideal prosthetic component positioning, corresponding to group 1 of patients, and ii) the $3^{\circ}, 5^{\circ}$ and $8^{\circ}$ of varus tibial malposition, but with a balanced knee, corresponding to patients in group 2. Malposition in varus for the tibial component results from extensive surgical removal from the medial part and little removal from the lateral part of the tibial plateau. The in vivo kinematics tests for the knee, have been achieved using SIMI Motion (SIMI Reality Motion Systems $\mathrm{GmbH}$ ), a performance system for image acquisition and image analysis. The image acquisition was achieved with two video cameras, high speed, in two planes at a $90^{\circ}$ angle. The metal implants were made of cobalt chromium molybdenum alloy (CoCrMo). The femoral component wasentirely made of CoCrMo alloy. The tibial component consisted of two parts. One was made of CoCrMo alloy, and the other of PE. The elastic modulus of the material (polyethylene) was established at 1,016 MPa, and the Poisson constant considered 0.46 . The modulus of elasticity was obtained by the ratio between stress per area unit and the consecutive deformation.

Statistical analysis. SPSS software 20 (IBM Corp.) was used for statistical analysis. $\mathrm{P}<0.05$ was assigned to indicate statistical significance. The results are expressed as the value of the standard deviation \pm the arithmetic mean for continuous quantitative variables. Quantitative variables were expressed as proportions. The independent t-test was performed to determine statistical significance. We considered the results to be statistically significant if the P-values concerning the two groups were $<0.05$, with a $95 \%$ confidence interval.

\section{Results}

We found no statistically significant differences between the group 1 and 2 regarding demographic characteristics: Age, sex, height and weight. In addition, we noted no differences in terms of postoperative evolution of KSS, frontal laxity and range of motion (Table I).

However, we did observe a statistically significant difference between the groups, regarding the positioning of the tibial component. The mean angle between the tibial component and the tibial mechanical axis was $1 \pm 3.5^{\circ}$ of varus for group 1 , and respectively $3 \pm 3.5^{\circ}$ of varus for group $2(\mathrm{P}<0.001)$. We did not need any revision for any of the operated knees at the final follow-up period. The mean follow-up interval was 6 years. The results of the finite element analysis are summarized in Table II.

We also measured the stress around the tibial post for $3^{\circ}, 5^{\circ}$ and $8^{\circ}$ of tibial varus malalignment (Fig. 1).

For $3^{\circ}$, the maximum contact pressure (13.7 MPa) was around the base of the tibial post, especially on the lateral side. Towards the tip of the post there was a decrease in pressure: 5.4 $\mathrm{MPa}$ on the lateral side (L) and $4 \mathrm{MPa}$ on the medial (M) side. For $5^{\circ}$, the maximum contact pressure increased to $17.5 \mathrm{MPa}$ around the post base. At the top of the post, the contact pressure was $8 \mathrm{MPa}$ placed on the lateral part and $3.5 \mathrm{MPa}$ on the medial part. For $8^{\circ}$, the maximum contact pressure increased to $22.5 \mathrm{MPa}$ around the post base. At the top of the post, the contact pressure was $6.5 \mathrm{MPa}$ on the medial side of the tibial plateau and 14.1 MPa on the lateral side.

\section{Discussion}

If the contact pressure areas are known, it is possible to provide the potential wear of polyethylene (PE). The higher the contact pressure on the tibial component, the more severe the damage to the implant may be, due to particle disease (6). Polyethylene is a polymer which contains $\mathrm{CH}_{2}$-chains $(8,9)$. For orthopedic implants, a type of this polymer is used, ultra-high molecular weight (UHMWPE). The elastic modulus of PE diminishes 
Table I. Postoperative outcomes.

\begin{tabular}{|c|c|c|c|c|}
\hline \multirow[b]{2}{*}{ Score } & \multicolumn{2}{|c|}{ Group $1(\mathrm{P}<0.001)$} & \multicolumn{2}{|c|}{ Group $2(\mathrm{P}<0.001)$} \\
\hline & Preoperative & Postoperative & Preoperative & Postoperative \\
\hline Knee society score & $25.15 \pm 12.1$ & $96.3 \pm 5.6$ & $20.45 \pm 10.1$ & $92.15 \pm 12.1$ \\
\hline Range of motion & $76.4 \pm 24.3^{\circ}$ & $110.3 \pm 9.7^{\circ}$ & $70.5 \pm 20.8^{\circ}$ & $100.4 \pm 20.3^{\circ}$ \\
\hline Frontal laxity & $8.81 \pm 2.8^{\circ}$ & $1.34 \pm 1.2^{\circ}$ & $10.5 \pm 4.8^{\circ}$ & $1.7 \pm 1.4^{\circ}$ \\
\hline
\end{tabular}

Table II. Finite element analysis.

\begin{tabular}{lccc}
\hline Alignment & $\begin{array}{c}\text { Maximum contact } \\
\text { pressure (megapascal) }\end{array}$ & $\begin{array}{c}\text { Mean contact pressure-medial } \\
\text { condyle (megapascal) }\end{array}$ & $\begin{array}{c}\text { Mean contact pressure-lateral } \\
\text { condyle (megapascal) }\end{array}$ \\
\hline Mechanical & 10.90 & 6.00 & 6.00 \\
$3^{\circ}$ of varus & 12.10 & 6.26 & 5.00 \\
$5^{\circ}$ of varus & 16.30 & 14.00 & 7.00 \\
$8^{\circ}$ of varus & 18.30 & 16.00 & 9.00 \\
\hline
\end{tabular}
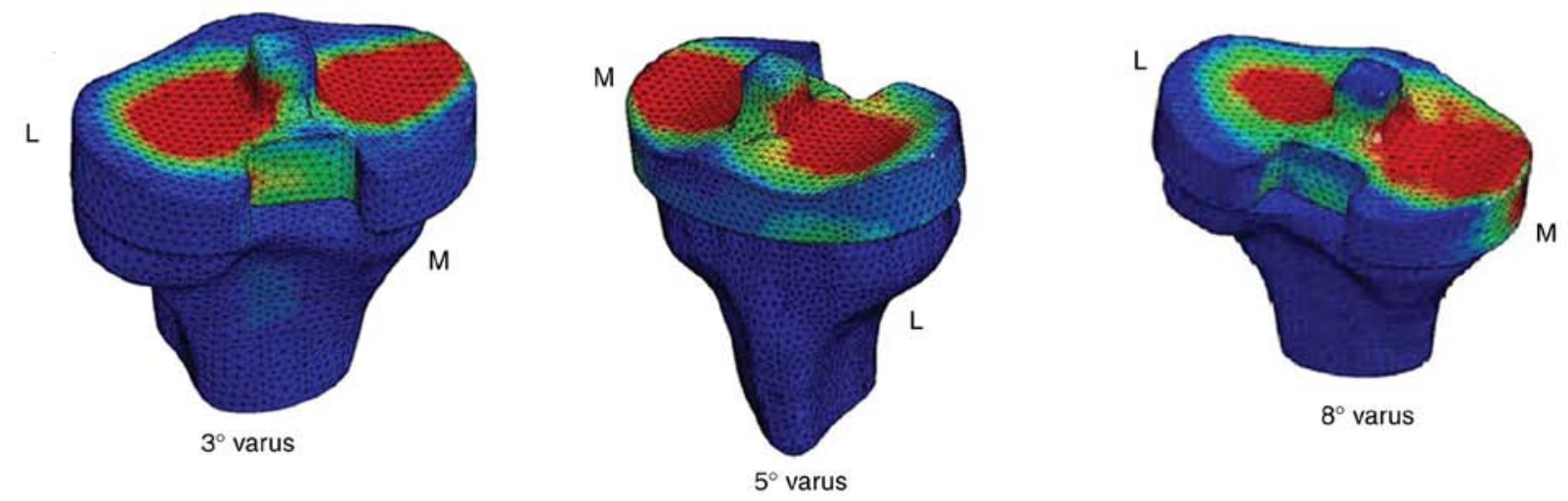

Figure 1. Stress around the tibial post. L, lateral side; M, medial side.

with tension. PE is a viscous-elastic implant, having the potential of plastic deformation in addition to wear. Beside wear, creep and plastic deformation may occur in PE fatigue. Long polymer chains of PE can slide over each other, resulting in creeping (10). Creep is responsible for delaminating, but decreases over time (10). The wear of PE results in particle debris between 0.2 to $10 \mu \mathrm{m}$ which results in phagocytosis by macrophages. The macrophages activate other mediators responsible for osteolysis such as tumor necrosis factor (TNF) $\alpha$, interleukin (IL)-1, and IL-6, or act like osteoclasts with direct bone resorption (10). A proper position of the implant is crucial for prosthetic joint load distribution, wear and survival of the prosthesis (6). The best placement of the prosthetic knee implant components remains uncertain. The disagreement among orthopedic surgeons is magnified by the important number of patients displeased with TKA. Most surgeons have concluded that a good alignment provides the best chances for survivorship of TKA (6). Mechanical best alignment represents the condition in which both angles between the femoral cut and the mechanical axis of the femur, respectively, between the tibial cut and the mechanical axis of the tibia have $90^{\circ}$ (11). However, some authors show that a femoral component placed in a $7^{\circ}$ valgus position, with the tibial plateau perpendicular to the long axis of the tibia, ensures an equal distribution of forces between the medial and the lateral sides and in consequence, assuring the best chances for survivorship (12). Howell et al considers that kinematic knee alignment is provided by aligning the transverse femoral component axis with the primary transverse axis in the femur about which the tibia makes movement of flexion and extension. The authors also consider, regarding the tibial component, that the longitudinal tibia axis should make a $90^{\circ}$ angle with the transverse femoral axis, about which flexion and extension of the tibia take place (13). For this purpose, the femoral cut should be made $1^{\circ}-2^{\circ}$ more valgus, while the tibial cut should be made $1^{\circ}-2^{\circ}$ varus, compared to the mechanically aligned TKA (14). Surgeons who support this approach claim that the restoration of mechanical alignment becomes improper in patients with constitutional alignment in varus or valgus and could cause increased tension in the collateral ligaments (15). They also argue that patients whose previous alignment is restored, may have better clinical and functional 
status than patients whose alignment is set to neutral (16). Irrespective of the operatory technique for TKA, an important problem for surgeons is represented by technical details. For example, instrument errors are of great consequence in this respect. For the femoral distal cut, precision depends on the intramedullar rod engaging the isthmus of the medullar canal for realign the anatomical axis. The precision of placement is influenced by the rod length and thickness, the diameter of the medullar canal, and by the placement of the entry hole for the intramedullar guides $(13,14)$. Therefore, surgeons aiming for mechanical alignment must be aware that instruments and the location of the entry holes could lead to large errors. The goal of our TKAs in both groups was to obtain mechanical alignment. With regard to tibial component alignment, we observed a significant difference between the two groups. In group 1, there was a neutral alignment $\left(1 \pm 3.5^{\circ}\right)$, while in group 2 it was in principle in the varus position $\left(3 \pm 3.5^{\circ}\right)$. The vast majority of patients in group 2 constituted extreme situations, because of additional asymmetric tibial varus cut. The finite element analysis showed that in a $3^{\circ}$ varus inclination of the interline, but with a balanced knee, the maximum contact pressure measured at the surface of tibial plateau increased by $11 \%$ compared to the value of mechanical alignment. At $8^{\circ}$ of inclination the maximum contact stress intensified by $68 \%$, even for a balanced knee. For $3^{\circ}$ of varus the distribution of forces was uniform on both tibial condyles, and the mean medial contact stress was $1.26 \mathrm{MPa}$ higher than the lateral contact stress. At $8^{\circ}$ of varus, we observed a medial displacement of the contact patch, and the mean medial contact stress was $7 \mathrm{MPa}$ higher than lateral stress. There are very few studies regarding post-cam stress of posterior-stabilized knee prosthesis. Pulosky et al investigated tibial post wear of certain types of knee prosthesis and found increased wear of the posterior part of the tibial post (17). Huang et al found a maximum von Mises contact stress of $21.2 \mathrm{MPa}$ at $60^{\circ}$ flexion and $27.6 \mathrm{MPa}$ at $150^{\circ}$ flexion (18). Koh et al researched post-cam design via finite element analysis to study the most normal-like knee mechanics (19). Amyand's hernia was a curious association in one case (20). Our study showed that for $3^{\circ}$ of varus inclination of the articular interline the maximum contact pressure was localized around the base of the tibial post, especially on the lateral side (13.7 MPa). As the inclination increased, the stress increased, also at the top of the post, thus leading to shearing and bending forces around the lateral and the medial aspect of the surface. These forces added to those from the posterior component of the post during flexion and contributed to tibial post wear.

In conclusion, polyethylene has a special abrasive resistance, shows low friction forces, high impact resistance, good chemical resistance and high energy absorption in contact with CoCrMo alloy. Still these advantages are also dependent on multiple factors such as surgical technique or implant positioning. The study findings support our technique for balancing severe varus knee and demonstrated that a computational model can be a useful tool for predicting clinical outcomes. If more than $3^{\circ}$ of varus in the tibia in the coronal plane, more stress will be sent in the post-cam and on the surface of the implant. Still further studies are necessary to completely evaluate the loadings, regarding complex 3D motion of the prosthetic knee and not only by varus-valgus stress.

\section{Acknowledgements}

Not applicable.

\section{Funding}

No funding was received.

\section{Availability of data and materials}

The datasets used and/or analyzed during the current study are available from the corresponding author on reasonable request.

\section{Authors' contributions}

NG, GS and HO had major contributions in the conception of the study and writing the manuscript. BS, MCTD and NB analyzed and interpreted the patient data and searched the literature for similar work and articles and contributed to writing the manuscript. All authors read and approved the final manuscript.

\section{Ethics approval and consent to participate}

The study was conducted according to the World Medical Association Declaration of Helsinki, using a protocol approved by the local Bioethics Committee from Elias Emergency Clinical Hospital (Bucharest, Romania). All patients previously signed an informed written consent concerning hospitalization, treatment and possible future publication of data.

\section{Patient consent for publication}

Not applicable.

\section{Competing interests}

The authors declare no conflict or competing interests.

\section{References}

1. Savlovschi C, Serban D, Andreescu Cv, Dascalu Am and Pantu H: Economic analysis of medical management applied for left colostomy. Chirurgia (Bucur) 108: 666-669, 2013.

2. Fang DM, Ritter MA and Davis KE: Coronal alignment in total knee arthroplasty: Just how important is it? J Arthroplasty 24: 39-43, 2009.

3. Berend ME, Ritter MA, Meding JB, Faris PM, Keating EM, Redelman R, Faris GW and Davis KE: Tibial component failure mechanisms in total knee arthroplasty. Clin Orthop Relat Res 428: 26-34, 2004.

4. Parratte S, Pagnano MW, Trousdale RT and Berry DJ: Effect of postoperative mechanical axis alignment on the fifteen-year survival of modern, cemented total knee replacements. J Bone Joint Surg Am 92: 2143-2149, 2010.

5. Schulze A and Scharf HP: Satisfaction after total knee arthroplasty: Comparison of 1990-1999 with 2000-2012. Orthopade 42: 858-865, 2013 (In German).

6. Dossett HG, Swartz GJ, Estrada NA, Lefevre GW and Kwasman BG: Kinematically versus mechanically aligned total knee arthroplasty. Orthopedics 35: e160-e169, 2012.

7. Insall JN, Dorr LD, Scott RD and Scott WN: Rationale of the knee society clinical rating system. Clin Orthop Relat Res 248: 13-14, 1989. 
8. Socea B, Socea LI, Bratu OG, Mastalier B, Dimitriu M, Carap A and Constantin VD: Recurrence rates and mesh shrinkage after polypropylene vs. Polyester mesh hernia repair in complicated hernias. Revista de Materiale Plastice 55: 79-81, 2018.

9. Socea B, Carâp A, Bratu OG, Diaconu CC, Dimitriu M, Socea LI, Bobic S and Constantin, VD: The role of the composite and biologic meshes in the trocar site hernia repair following laparoscopic surgery. Revista de Materiale Plastice 55: 146-148, 2018.

10. Bitar D and Parvizi J: Biological response to prosthetic debris. World J Orthop 6: 172-189, 2015

11. Insall JN, Binazzi R, Soudry M and Mestriner LA: Total knee arthroplasty. Clin Orthop Relat Res 192: 13-22, 1985.

12. Hsu RW, Himeno S, Coventry MB and Chao EY: Normal axial alignment of the lower extremity and load-bearing distribution at the knee. Clin Orthop Relat Res 255: 215-227, 1990.

13. Howell SM, Roth JD and Hull ML: Kinematic alignment in total knee arthroplasty. Definition, history, principle, surgical technique, and results of an alignment option for TKA. Arthropaedia 1: 44-53, 2014.

14. Howell SM and Hull ML: Kinematic alignment in total knee arthroplasty. In: Insall \& Scott Surgery of the Knee. Scott N (ed). 5th edition. Chapter 121. Elsevier-Churchill Livingstone, Philadelphia, pp1255-1268, 2012.
15. Gu Y, Roth JD, Howell SM and Hull ML: How frequently do four methods for mechanically aligning a total knee arthroplasty cause collateral ligament imbalance and change alignment from normal in white patients? J Bone Joint Surg Am 96: e101, 2014.

16. Vanlommel L, Vanlommel J, Claes S and Bellemans J: Slight undercorrection following total knee arthroplasty results in superior clinical outcomes in varus knees. Knee Surg Sports Traumatol Arthrosc 21: 2325-2330, 2013.

17. Puloski SK, McCalden RW, MacDonald SJ, Rorabeck $\mathrm{CH}$ and Bourne RB: Tibial post wear in posterior stabilized total knee arthroplasty: An unrecognized source of polyethylene debris. J Bone Joint Surg Am 83: 390-397, 2001.

18. Huang CH, Liau JJ, Cheng CK and Huang CH: Influence of post-cam design on stresses on posterior-stabilized tibial posts. Clin Orthop Relat Res 450: 150-156, 2006.

19. Koh YG, Son J, Kwon OR, Kwon SK and Kang KT: Effect of post-cam design for normal knee joint kinematic, ligament, and quadriceps force in patient-specific posterior-stabilized total kneearthroplasty by using finite element analysis. Biomed Res Int 2018: 2438980, 2018.

20. Savlovschi C, Brănescu C, Serban D, Tudor C, Găvan C, Shanabli A, Comandaşu M, Vasilescu L, Borcan R, Dumitrescu D, et al: Hernia Amyand-caz clinic [Amyand's hernia-a clinical case]. Chirurgia (Bucur) 105: 409-414, 2010. 